\title{
SurfaceOxidized Mesoporous Carbons Derived from Porous Silicon as Dual Polysulfide Confinement and Anchoring Cathodes in Lithium Sulfur Batteries
}

Rachel Carter ${ }^{1}$, Dennis Ejorh ${ }^{1}$, Keith Share ${ }^{1,2}$, Adam P. Cohn ${ }^{1}$, Anna Douglas ${ }^{1,2}$, Nitin Muralidharan ${ }^{1,2}$, Trenton M. Tovar ${ }^{3}$, and Cary L. Pint ${ }^{1,2, *}$

${ }^{1}$ Department of Mechanical Engineering, Vanderbilt University, Nashville, TN 37235, USA

${ }^{2}$ Interdisciplinary Materials Science Program, Vanderbilt University, Nashville, TN 37235, USA

${ }^{3}$ Department of Chemical and Biomolecular Engineering, Vanderbilt University, Nashville, TN 37235, USA

\section{Corresponding Author}

*Cary L. Pint, email: cary.l.pint@vanderbilt.edu 


\begin{abstract}
Despite widespreadfocus on porous carbons for lithium-sulfur battery cathode materials, electrode design to preserve mass-specific performance and sustainedextended cycling stability remains a challenge. Here, we demonstrateelectrochemically etched porous silicon as a sacrificial template to produce a new class of functionalmesoporous carbons optimized for dual chemical and physical confinement of soluble polysulfides in lithium-sulfur battery cathodes. Melt infiltration loading of sulfur at 60 wt.\% enables initial discharge capacity of 1350 $\mathrm{mAh} / \mathrm{g}_{\text {sulfurat }}$ rates of $0.1 \mathrm{C}$ - approaching theoretical capacity of $1675 \mathrm{mAh} / \mathrm{g}_{\text {sulfur. }}$ Cycling performance measured at $0.2 \mathrm{C}$ indicates $81 \%$ capacity retention measured over 100 cycles with 830mAh/gsulfur capacity. Unlike other carbons, this template combines structural properties necessary for sulfur containment and polysulfide confinement to achieve high specific capacity, but also boasts surface-bound oxygen-containing functional groups that are able to chemically anchor the soluble $\mathrm{Li}_{2} \mathrm{~S}_{\mathrm{n}}$ species on the interior of the mesoporous carbon to sustain cycling performance. In turn, this elucidates a scalable and competitive material framework that is capable, without the addition of additional membranes or inactive anchoring materials, to provide the simultaneous anchoring and confinement effects necessary to overcome performance limitations in lithium sulfur batteries.
\end{abstract}

Keywords:lithium sulfur battery, sulfur cathode, mesoporous carbon, porous silicon, polysulfide immobilization 


\section{Introduction}

Lithium sulfur batteries are poised as a technology to provide a midpoint between the energy storage capability of lithium-ion batteries, and emerging batteries that have promise to compete with the energy density of fossil fuels. This advance is critical due to the increasing reliance of modern technology on portable power storage.[1]The most primitive design of a lithium-sulfur battery incorporates an elemental sulfur cathode and lithium metal anode which exhibits a high theoretical energy density (2600Wh/kg[2]) with the inherent benefit of the low cost and earth abundance of sulfur. However, practical routes to achieve lithium-sulfur batteries based on complete conversion of elemental sulfur to lithium sulfide have proven to be quite challenging due to three major issues 1) large volumetric expansion 2) poor electrical conductivity and 3) polysulfide stability in electrolyte.[3] This has led to an immense interest in using carbon as a backbone material for cathodic sulfur loading since lightweight carbons can be produced with high porosity, carbons are natively conductive, and high sulfur loading of carbons can enable total electrode capacities that significantly surpass traditional lithium-ion cathodes.

Among the most studied materials are mesoporous carbons. Early studies by Ji et al.demonstrated a mesoporous carbon composite cathode by melt diffusing CMK-3 with 70 wt.\% S. The cathode was capable of delivering high capacity of $1320 \mathrm{mAh} / \mathrm{g}$, but with durability only analyzed over 20 cycles.[4]Subsequent efforts with mesoporous carbons exhibit high capacity with rapidlinear decay of storage capacity that has provided a bottleneck for these materials.[58]This capacity fade is attributed to polysulfide shuttling, where elemental sulfur (stored as $\mathrm{S}_{8}$ crystals) is reduced to the soluble $\mathrm{S}_{6}{ }^{2-}$ (bound to $2 \mathrm{Li}^{+}$)state during the multistep transitionto lithium sulfide. If out-diffusion of the soluble compound $\left(\mathrm{Li}_{2} \mathrm{~S}_{6}\right)$ is permitted from the cathode side, fouling of the lithium metal anode can occur leading to rapid loss of storage 
capability.[2]Whereas some attempts have been made to address this through electrolyte additives,[9]recent efforts have mostly been aimed toward materials-level approaches to mitigate these challenges.

The first of these approaches employs an interlayer between the cathode and separator to trap dissolved polysulfides near the cathode and instigate precipitation on the cathode before formation of the final discharge product $\mathrm{Li}_{2} \mathrm{~S} .[10,11]$ This strategy shows enhanced cycling performance but requires addition of mass and volume with the interlayer as added element in device configuration. Recent work has shown the ability to achieve high areal loadings of sulfur ( $>16 \mathrm{mg} / \mathrm{cm}^{2}$ ) with the use of this interlayer approach [12] although whether the use of interlayers can be competitive on the benchmark of mass-specific performance, which is the current standard benchmark of the battery industry, remains a challenge. Another strategy for preventing polysulfide dissolution is sulfur encapsulation using synthesized constructs such as yolk-shell structures,[13, 14] graphene wrapping,[15, 16] and microporous carbons.[5-7, 1726]Encapsulation in microporous carbon is most commonly carried out in carbide derived carbon materials due to the uniform micropore sizes $(<2 \mathrm{~nm})$ that arise based on removal of metal clusters from the carbide which are interconnected through mesoporous networks.[19]Building on this approach, recent efforts have proposed that confinement in ultra-small pores $(<0.5 \mathrm{~nm})$ can prevent the formation of soluble $\mathrm{Li}_{2} \mathrm{~S}_{6}$ [27-30] which can improve performance. Most recently, anchoring materials with polar surface characteristics such as metal oxides[31-34] and functionalized carbon materials[35, 36] have been demonstrated to pin soluble polysulfide species to the cathode and reduce capacity fade.[26] However this requires use of heavy metal oxides resulting in low sulfur loading or expensive material processing.Overall, current efforts therefore build upon either structural or chemical roles of confinement in sulfur cathode 
materials, leading to an emerging research area that can simultaneously combine these confinement strategies into the framework of a single electrode material.

In this spirit, our work demonstrates a new family of mesoporous carbon materials that are derived from porous silicon and unlike other reports on mesoporous carbons,simultaneously exhibit both structural confinement and chemical anchoring of soluble polysulfides for lithium sulfur batteries. Defective carbons are directly grown in a self-limiting manner onto porous silicon templates, and the porous silicon is dissolved in aqueous basic (salt-water) conditions to yield mesoporous carbons with structural features correlated to the porous silicon template, and surface chemical features dictated by the material synthesis.Unlike other routes to produce mesoporous carbons such as carbide-derived carbons, our work enables a route to decouple the carbon chemical properties and the structural properties in the framework of scalable or low-cost processing based on conventional semiconductor manufacturing techniques. In this manner, low-cost and scalable silicon processing can be extended to form templates that can be thermally treated with hydrocarbons (in parallel to combustion processes used to make cheap carbons such as carbon black) and rapidly dissolved in water. Unlike carbide-derived carbons, this provides a true templating approach due to the self-limited growth of carbon on the template, and highly tunable nano- and microscale features building upon over five decades of demonstrated structural control of porous silicon fabrication, which is more versatile than the processing of micron sized spherical particles possessing mesopores used for other mesoporous carbons.[4, 6, 19, 20].We demonstrate high initial capacity and excellent capacity retention of these cathode materials approaching key cathodic performance targets thatarises from a two-fold strategy to inhibit polysulfide shuttling. 


\section{Experimental}

\subsection{Sample Preparation}

\subsubsection{Porous Carbon Preparation}

Porous silicon was formed through electrochemical etch of highly boron doped $\left(\mathrm{p}^{++}\right)$silicon wafers using an AMMT porous silicon etching system. The etch applied was $60 \mathrm{~mA} / \mathrm{cm}^{2}$ current density to the wafer for 100 seconds in $8: 3$ (v:v) ethanol, hydrofluoric acid (50\% water by volume Sigma Aldrich) electrolyte. The wafer was then fully rinsed in ethanol and then loaded into a Lindberg Blue 1” tube furnace for carbon growth by chemical vapor deposition (CVD). The furnace was ramped to $650^{\circ}$ ander 1 SLM of Ar and 200 SCCM of $\mathrm{H}_{2 .}$. Then 10 SCCM of $\mathrm{C}_{2} \mathrm{H}_{4}$ is allowed to flow as the furnace ramps to $750^{\circ} \mathrm{CUpon}$ reaching $750^{\circ}$ Che temperature is maintained for 10 minutes and then ramped to $850^{\circ} \mathrm{Cand}$ held for 10 minutes. The acetylene was then turned off and the system is allowed to cool to room temperature under hydrogen and argon. After removing the sample from the tube furnace it is heated to $60^{\circ} \mathrm{Gn}$ a $2 \mathrm{M}$ solution of $\mathrm{NaOH}$ for 48 hours. The freestanding carbon flakes are washed in nanopure water while monitoring $\mathrm{pH}$ until the solution is neutral. The material are allowed to rest in a water bath for 1 hour and exchanged once. The water is then removed and material suspended in ethanol. The carbon and ethanol solution is probe sonicated for 2 minutes to form uniformly sized particulates. The solution is centrifuged for 15 minutes at $9000 \mathrm{rpm}$ and the ethanol is removed leaving micron scale mesoporous carbon flakes in powder form.

\subsubsection{Porous Carbon/Sulfur Composite Preparation}

This powder is then mixed with elemental sulfur in a 1:3 ratio and heated just above the melting point of sulfur $120^{\circ}$ Cfor 6 hours. The carbon is fully infiltrated through capillary action 
and low surface energy of sulfur. The mixture is then flash heated to $200^{\circ}$ Gor 5 minutes to burn off the excess sulfur not infiltrated into the carbon.

\subsection{Cell Fabrication and Electrochemical Measurement}

The material was then prepared into electrodes by mixing the prepared material with PVDF and carbon black in a 7:1.5:1.5 ratio by mass with a small amount of NMP (Sigma Aldrich). The mixture was bath sonicated for 1 hour and then applied to steel discs (MTI) and vacuum dried. This process resulted in an areal sulfur loading of $0.3-0.6 \mathrm{mg} / \mathrm{cm}^{2}$. Electrochemical half-cells were assembled in an argon glovebox using CR 2032 stainless steel coin cells (MTI) with prepared electrode as cathode, elemental lithium as counter and reference electrode and 2500 Celgard as separator wetted with electrolyte. The electrolyte used was $1 \mathrm{M} \mathrm{LiTFSi,} 0.25 \mathrm{M} \mathrm{LiNO}$ in DME:DOL 1:1 by volume (all from Sigma Aldrich).Electrochemical testing was carried out using Metrohm Autolab and MTI potentiostats.

\subsection{Materials Characterization}

Materials Characterization was carried out using SEM (Zeiss), TEM (FEI) to investigate surface

morphology and composite features. Raman (Raith) spectroscopy was used with $532 \mathrm{~nm}$ laser excitation to assess carbon quality and surface features and XRD was used to assess crystallinity. BET (ASAP 2020 V3.01 H) was employed to analyze the pore characteristics, and UV/Vis (Cary) was employed to verify polysulfide immobilization through polar binding.

\section{Results and Discussion}

The process used to develop porous silicon derived mesoporous carbon is shown schematically in Fig. 1a.This process builds on our previous works in which carbon passivated porous silicon was studied[37, 38] and employed as supercapacitor electrodes,[39] Li-ion battery anodes[40] and photocapacitor electrodes.[40]The first step of the process is an electrochemical 
etch of silicon to generate a controlled, highly porous template. Next a thin conformal layer of defective carbon is grown on the porous silicon template. Since porous silicon is rapidly dissolved in aqueous, basic solution,[38] the mesoporous carbon is released from the porous silicon in a manner that allows the carbon to retain the pore network and structure of the porous silicon sacrificial template. Fig. S1 shows a photograph of freestanding porous-silicon derived carbon layers fully intact upon releasing from the silicon. These macro-scale pieces are then sonicated and dried to yield a powder of micron-scale particulates that can be effectively loaded with sulfur.

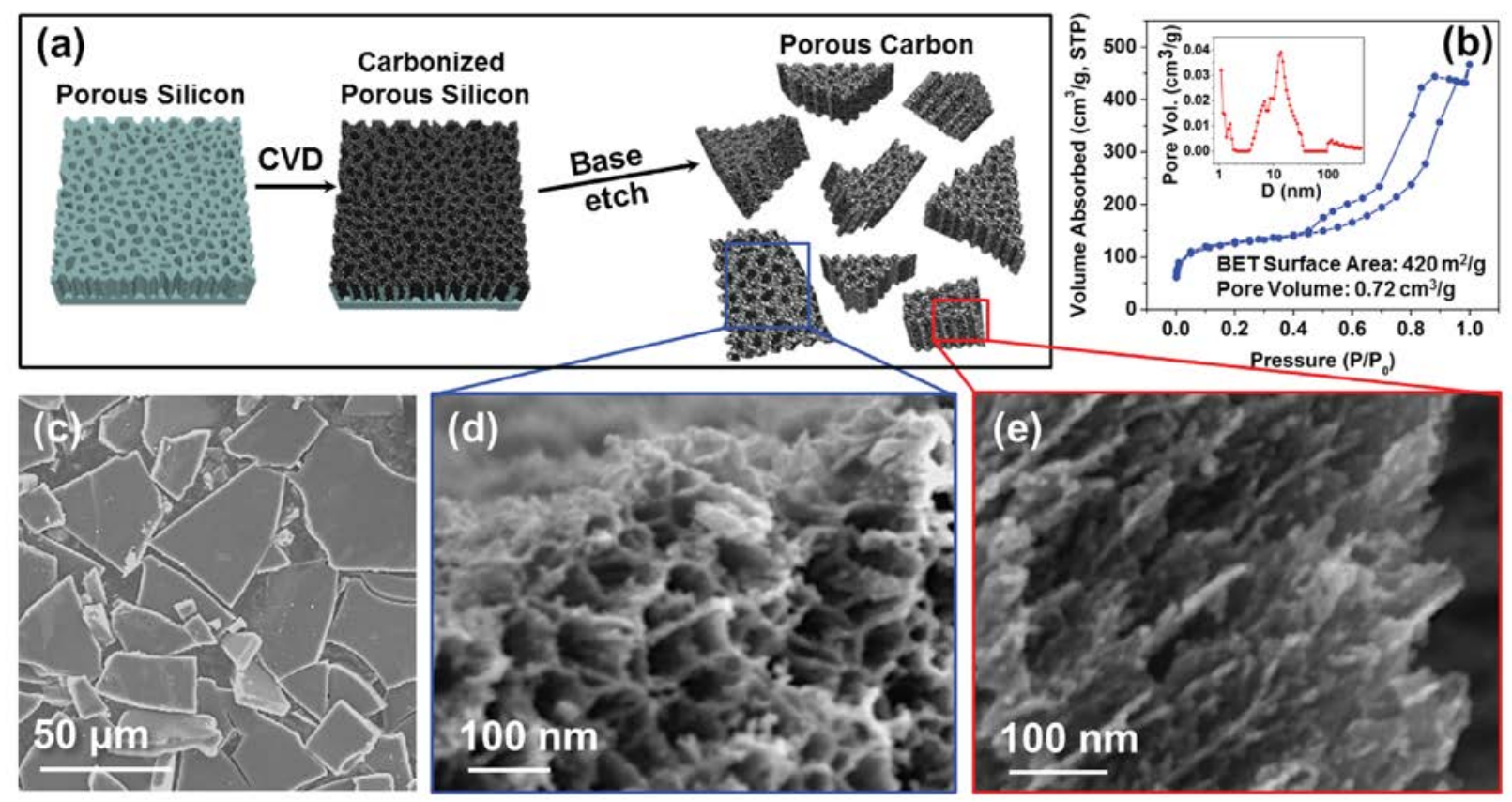

Fig. 1. (a) Schematic representation of mesoporous carbon material processing, (b), Nitrogen adsorption/desorption isotherms with inset showing pore size distribution (c) scanning electron micrograph of sonicated porous carbon, leaving micron scale flakes of interconnected material, (d) SEM top down view of mesoporous carbon material, (e) SEM cross sectional view of mesporous carbon. 
This process produces a high porosity, low-density porous carbon material. To assess these properties in the framework of sulfur cathodes, nitrogen adsorption-desorption isotherms were used to determine surface area and pore volume. The isotherm shape (Fig. 1b) reveals type IV hysteresis according to the IUPAC classification[15] indicating mesoporous character.[21] The Brunauer-Emmett-Teller (BET) surface area measured from the isotherm was $420 \mathrm{~m}^{2} / \mathrm{g}$ and pore volume determined using the Horvath-Kawazoe model was $0.72 \mathrm{~cm}^{3} / \mathrm{g}$, with a majority of mesopore volume $\left(0.60 \mathrm{~cm}^{3} / \mathrm{g}\right.$ in mesoporous range and $0.11 \mathrm{~cm}^{3} / \mathrm{g}$ in microporous range). This high pore volume concentrated in the mesoporous range will enable ideal behavior in a sulfur anchoring material.[21, 24]Similarly, the pore size distribution (Figure 1b, inset) reveals a broad peak ranging from $3.7-37 \mathrm{~nm}$, centered at $13.7 \mathrm{~nm}$, and a peak near $\sim 1 \mathrm{~nm}$. This correlates with to the structure of porous silicon that contains pore channels linked to pockets of micropores in the materials (apparent in SEM images Fig. 1e-f).Scanning electron microscope (SEM) analysis of these microscale mesoporous carbon particulates is shown inFig. 1c, with higher magnification images showing the nanoscale pore features in Fig. 1d and e. From SEM the high porosity of the material is apparent with a pore diameter of about15 nm (Fig. 1d) which agrees well with the pore size distribution obtained through BET. The images also revealthe thin, semitransparent flake-like edges (Fig. 1e) similar to other graphenic materials. Fig. 1e also reveals the tree-like porous structure, characteristic of the porous silicon template(see Fig. S2), further emphasizing the material maintains the structural integrity of the template following removal. Additional SEM images of the porous carbon material are provided in the Supporting Information (Fig. S3).

To prepare a lithium-sulfur battery cathode, the carbon powders are melt infiltrated with sulfur via a simple method commonly used to produce carbon-sulfur composites.[27, 30, 41] Fig. 
2 shows SEM images both before (Fig. 2a) and after (Fig. 2b) sulfur infiltration to 60wt.\% sulfur. Sulfur loading is measured to be $\sim 0.5 \mathrm{mg} / \mathrm{cm}^{2}$ and $\sim 0.3 \mu \mathrm{g} / \mathrm{cm}^{2}$ relative to total footprint and specific surface area, respectively. In our case, the areal loading is limited by the drop cast

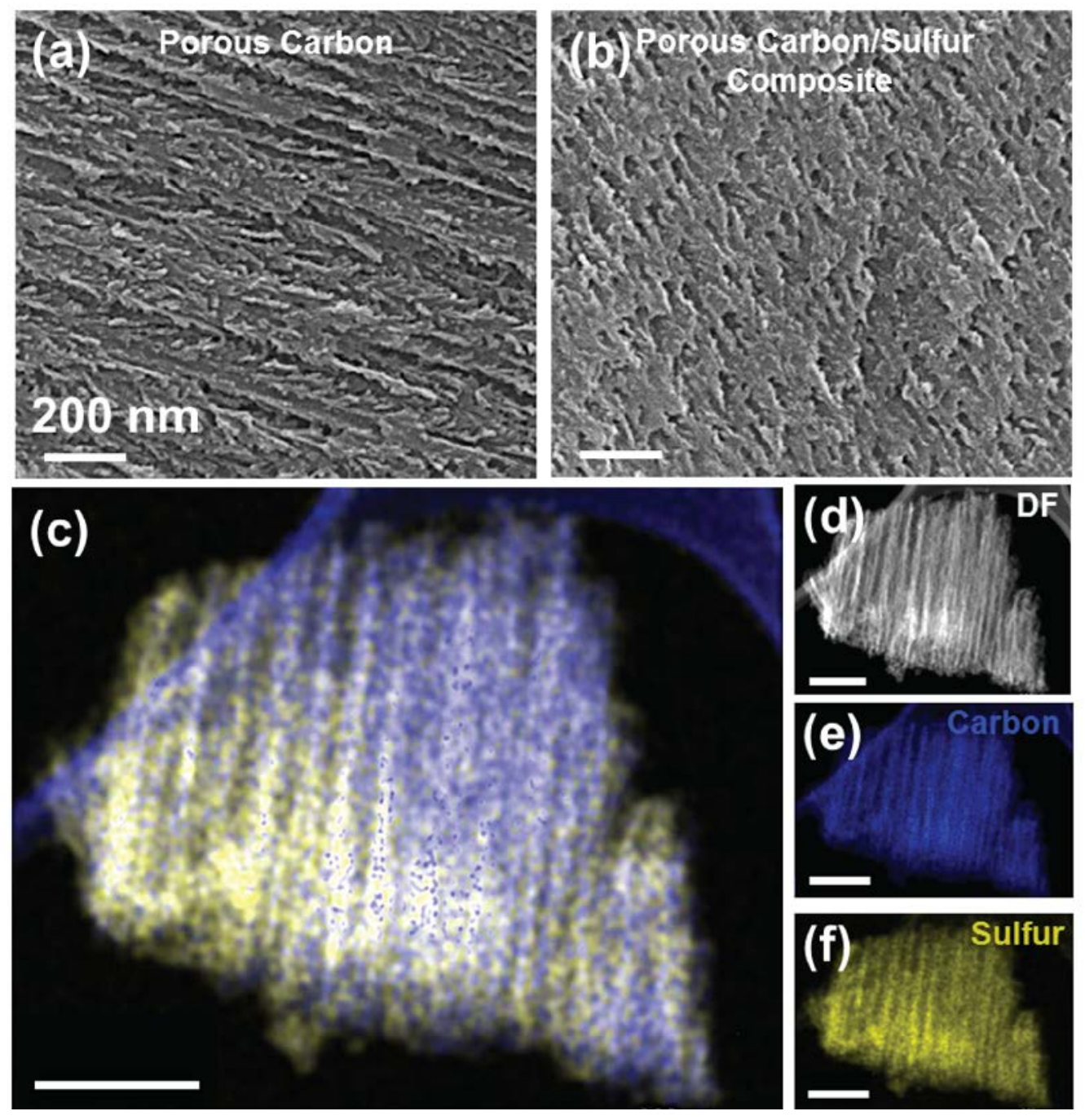

Fig. 2. (a) SEM cross sectional view of mesoporous carbon material, (b) SEM cross sectional view of mesoporous carbon/sulfur composite material (c) STEM EDS composite map (d) STEM image of a mesoporous carbon flake, (e-f). STEM elemental map (e) of carbon and (e) of sulfur. All scale bars are $200 \mathrm{~nm}$. 
method used to fabricate electrodes, but notably the electrode material exhibits functional nanoscale tree-like architectures embedded in microscale thickness structures that are well-suited for thick electrodes due to the storage of sulfur near electrically addressable carbon sites and the large channels that extend throughout the material. Whereas areal loading is important to minimize packaging weight in a commercial cell architecture, lithium-sulfur batteries used to replace current Li-ion technology must be rooted in excellent specific performance [42], motivating our focus on the mass-specific properties of the battery. The material also maintains a high level of porosity even with the relatively high loading of sulfur that gives promise for excellent electrolyte penetration and good performance. To verify the distribution of sulfur in the porous carbon, a flake of porous carbon/sulfur compositewas imaged using scanning transmission electron microscopy (STEM) for elemental mapping (Fig. 2c). Elemental maps of carbon and sulfur (Figs 2e, 2e) indicate uniform distribution of sulfur in the porous network of the carbon. The corresponding X-ray signature is found in Fig. S4.Thermogravimetric Analysis (TGA) was further used to verify the sulfur loading of the material and is shown in Fig. S5. This analysis was used to normalize device data to the mass of sulfur present in order to compare to the theoretical device performance.

In order to further analyze the porous carbon/sulfur composite, Raman spectroscopywas used to compare the porous carbon and the porous carbon/sulfur composite. The porous carbon exhibited characteristic D and G carbon peaks representing defective $\mathrm{sp}^{3}$ hybridized carbon bonds (1337 $\left.\mathrm{cm}^{-1}\right)$ and ideal in-plane $\mathrm{sp}^{2}$ carbon bonds $\left(1594 \mathrm{~cm}^{-1}\right)$ respectively (Fig. 3a). Upon sulfur infiltration, the Raman signature of elemental sulfur $\left(S_{8}\right)$ is most prominent at 219 and 473 $\mathrm{cm}^{-1}$ and is indicative of the presence of highly crystalline, $\mathrm{S}_{8}$, sulfur stored on the surface of the low in crystallinity, defective, porous carbon. Following sulfur infiltration into the carbon, both 

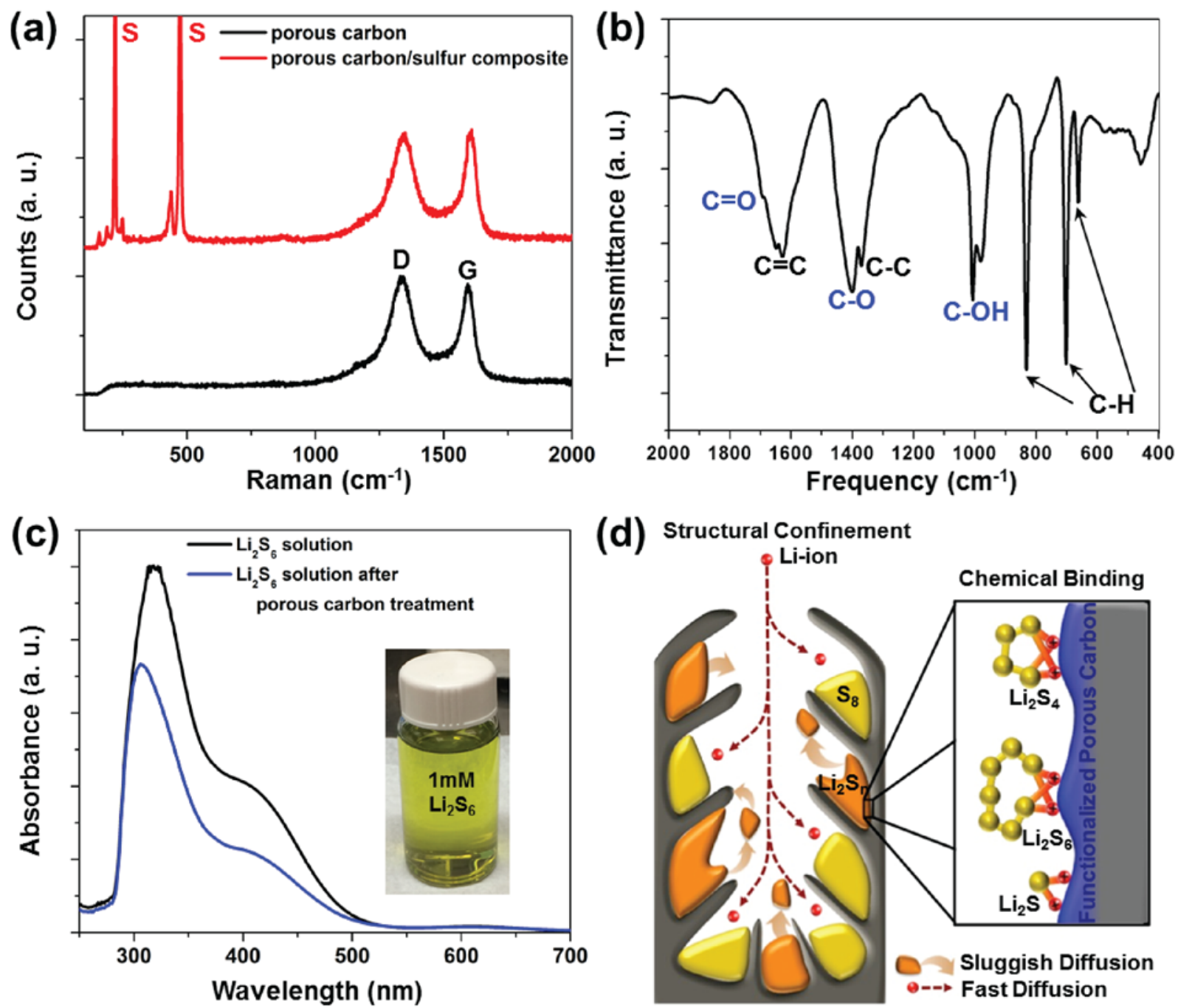

Fig. 3. (a) Raman spectroscopy with $532 \mathrm{~nm}$ excitation of porous silicon derived mesoporous carbon and mesoporous carbon/sulfur composite material, (b) Fourier transform infrared spectroscopy of mesoporous carbon material with notable stretch modes labeled, (c) UV/vis absorption spectra of $\mathrm{Li}_{2} \mathrm{~S}_{6}$ solution before and after exposure to porous carbon material with inset photo of polysulfide solution (d) schematic representation of two-fold polysulfide confinement by controlled pore structure (left) and by surface anchoring of polysulfides (right). 
the D and G peaks red shift, and corresponding Lorentzian fits to the D peak indicate peak broadening as emphasized by a full-width half maximum that increases from $241 \mathrm{~cm}^{-1}$ to $245 \mathrm{~cm}^{-}$ 1. In accordance with previous studies that report a similar effect, [26, 43] this also leads to a slight intensity change of the observed D peak. X-ray Diffraction (XRD) scans of the material (Fig. S6) present a similar result in that the defective and poorly crystalline nature of the porous silicon derived carbon - which is beneficial for Li-S cathodes, exhibits no prominent diffraction peaks in comparison to the strong crystalline $\mathrm{S}_{8}$ response for the composite material.

A key feature of this material is the combination of high defect concentration that leads to functionalized $\mathrm{sp}^{3}$ carbon surface sites and the controllable and interconnected porous structure that enables electrical conduction and sulfur storage.Recent studies have indicated that surface polar sites are critical to anchor soluble polysulfide species, and we elucidate this as a strength of this material fabrication approach. The $\mathrm{sp}^{3}$ hybridization of carbon that arises due to defects in the carbon lattice can be controlled during synthesis of this porous carbon material and leads to out-of-plane dangling bonds on the carbon surface that are susceptible to functionalization. Following the aqueous dissolution of the porous silicon template, the high defect content of the carbon is expected to lead to an oxidized surface that will anchor soluble polysulfides in accordance with recent reports. ${ }^{36}$ To study this, Fourier transform infrared spectroscopy (FTIR) spectroscopy is used to analyze the infrared response of a $\mathrm{KBr}$ pellet with porous carbon in a $1 \%$ mass ratio. The presence of $\mathrm{C}-\mathrm{OH}, \mathrm{C}-\mathrm{O}$, and $\mathrm{C}=\mathrm{O}$ groups are confirmed using FTIR (Fig. 3b). The three sharp peaks at lower frequencies $\left(663,702\right.$, and $831 \mathrm{~cm}^{-1}$ ) are C-H stretch modes, the peak at $977 \mathrm{~cm}^{-1}$ agrees well with a bound O-H group stretch, and the sharp peak at $1007 \mathrm{~cm}^{-1}$ reveals C-O stretch with the bound $\mathrm{OH}$. The double peaks in range of $1350-1450 \mathrm{~cm}^{-1}$ agree well with C-C and C-O stretching respectively and the broad peak from 1550-1750 $\mathrm{cm}^{-1}$ comprises of 
$\mathrm{C}=\mathrm{C}$ and $\mathrm{C}=\mathrm{O}$ stretch with the $\mathrm{C}=\mathrm{O}$ arising in the shoulder at the higher wavenumbers of the peak.[44] This is representative of a material with a high defect contentand polar oxygencontaining functional species on the surface. These polar groups provide pinning sites for the polar $2 \mathrm{Li}^{+}$species in soluble $\mathrm{Li}_{2} \mathrm{~S}_{\mathrm{n}}$ compounds to mitigate soluble species dissolution into the electrolyte. With the verification of surface oxides and defects through Raman and FTIR, further efforts were carried out to support the idea that charge transfer was not limited by the conduction properties of the porous carbon materials. Through-plane I-V measurements were carried out and electrical conductivity following full processing of the porous carbon was calculated to be 16.4 $\Omega^{-1} \mathrm{~cm}^{-1}$ (supplementary information, Figure S7). This reflects a slight enhancement in conductivity in comparison to other carbide derived carbons and carbon black materials, $[45$, 46]even with the surface oxidation present in the material. This supports the principle of electron-accessible pathways in the carbon electrode to sustain electrochemical conversion of sulfur while simultaneously providing anchoring sites that support efficient transformation to insoluble solid products.

To demonstrate the polar binding and anchoring effect of these materials, UV/vis absorption spectra was used to compare the known $\mathrm{Li}_{2} \mathrm{~S}_{6}$ absorption peaks[47] before and after exposure to porous silicon derived mesporous carbon. Since the polysulfide discharge product $\mathrm{Li}_{2} \mathrm{~S}_{6}$ is soluble in DME:DOL, fully solubilized solutions of the species were developed by adding stoichiometric amounts of $\mathrm{Li}_{2} \mathrm{~S}$ and $\mathrm{S}_{8}$ to the solvents and spinning the solutions at $60^{\circ} \mathrm{C}$ for 12 hours. Porous carbon was then added to the solution of known concentration and allowed to spin for 24 hours. The spectrum of this solution was compared to solution not treated with carbon (Fig. 3c). The porous carbon treated solution exhibited an absorption intensity decrease at the known $\mathrm{S}_{6}{ }^{2-}$ absorption peaks around 300 and 350 indicating a change of concentration of 
these species and verifying the ability of the material to bind the polar and soluble discharge product.[31, 48] The peaks also exhibit a blue shift, which is another sign of strong interaction between the material and the solution, resulting in slight change in behavior of still solubilized species. To further verify the anchoring of polar polysulfides on the surface oxidized carbons, the porous carbon formed after spinning for 24 hours was placed in an airtight cell with a glass window, and analyzed using Raman spectroscopy to mitigate adverse effects of air exposure on analysis of this interface. Both the $\mathrm{D}$ and $\mathrm{G}$ carbon peaks exhibit red shifts of $\sim 10 \mathrm{~cm}^{-1}$ that emphasizes the presence of a strong surface interaction between the soluble $\mathrm{Li}_{2} \mathrm{~S}_{6}$ and the porous carbon surface (Fig. S8). Further, additional tests were carried out to examine the role of physically absorbed polysulfides on observations in Fig. 2C. To examine this, we carried out an identical test using single-walled carbon nanotubes (SWCNTs) which exhibit significantly higher surface area (over 2x) than our mesoporous carbon but with no oxidized surface species that would promote polar binding. As evident in Fig. S9, the UV-Vis spectra with the SWCNTs shows virtually no change in the absorption of the solution, ruling out physical absorption as playing a role in this data. In turn, these control experiments provide robust mechanistic support elucidating the utility of polar anchoring sites in the cathode material.

Fig. 3d schematically represents the utility of this material for confining soluble polysulfides in the framework of lithium-sulfur cathodes. First, structural confinement is a result of the ideal material geometry provided by the porous silicon template. The high aspect ratio of nanopores (about $15 \mathrm{~nm}$ in diameter and $8 \mu \mathrm{m}$ in depth) provides a wide pathway for the rapid transport of $\mathrm{Li}^{+}$species $(0.18 \mathrm{~nm})$, but a torturous pathway for the transport of soluble polysulfides. Along the pore channels are small micropores that provide locally electrically interconnected binding sites for ideal confinement in a sulfur cathode. Second, the confirmation 
of polar functional groups on the porous carbon surface enables chemical anchoring of soluble polysulfides in a manner that is synergistic with structural confining features. As discussed previously, dissolution (and capacity fade) occurs when $\mathrm{S}_{6}{ }^{2-}$ is not anchored to electrode materialsbecause of its solubility in DME:DOL electrolytes (most common for Li-S batteries). The binding of $\mathrm{Li}_{2} \mathrm{~S}_{8}$ to electron rich oxygen groups found on the carbon surface will allow for transition to $\mathrm{Li}_{2} \mathrm{~S}_{6}$ and then $\mathrm{Li}_{2} \mathrm{~S}_{4}$ without the dissolution of sulfur-containing species.The structure of several polysulfides is depicted in Fig. 3d to emphasize the strong polarity of the compounds and their binding on the functionalized carbon surface. The combination of these two effects, structural confinement and chemical binding, allow optimal characteristics expected for a sulfur cathode material and near-theoretical capacity since the active material is highly accessible and fully intact.It also allows strong cycling performance due to the prevention of polysulfide dissolution in subsequent cycles.

To support the picture in Fig. 3, full electrochemical characterization was carried out and is shown in Fig. 4. The material was galvanostatically charged and discharged at a rate of $0.1 \mathrm{C}$ (1675 mA/g $\left.\mathrm{g}_{\text {sulfur }}\right)$ to assess discharge capacity and durability. The material exhibited high initial discharge capacity of $1350 \mathrm{mAh} / \mathrm{g}_{\text {sulfur }}$ or $810 \mathrm{mAh} / \mathrm{g}_{\text {active }}$, the latter of which includes the mass of the porous carbon (Fig. 4a). This high initial discharge capacity approaches the theoretical capacity of lithium storage in sulfur. The strong stability of the upper plateau in which soluble polysulfides are formed confirms successful polysulfide confinement. The ratio of lower order polysulfides (LOPS) produced to higher order polysulfides (HOPs) on the second discharge was 1.6, which is competitive with recent state-of-the-art advances, but also indicates improved surface area for binding could enhance the formation of LOPS and further improve cycling performance. [49] This ratio slowly fades with cycling (1.24 at cycle 5) indicating lessened 


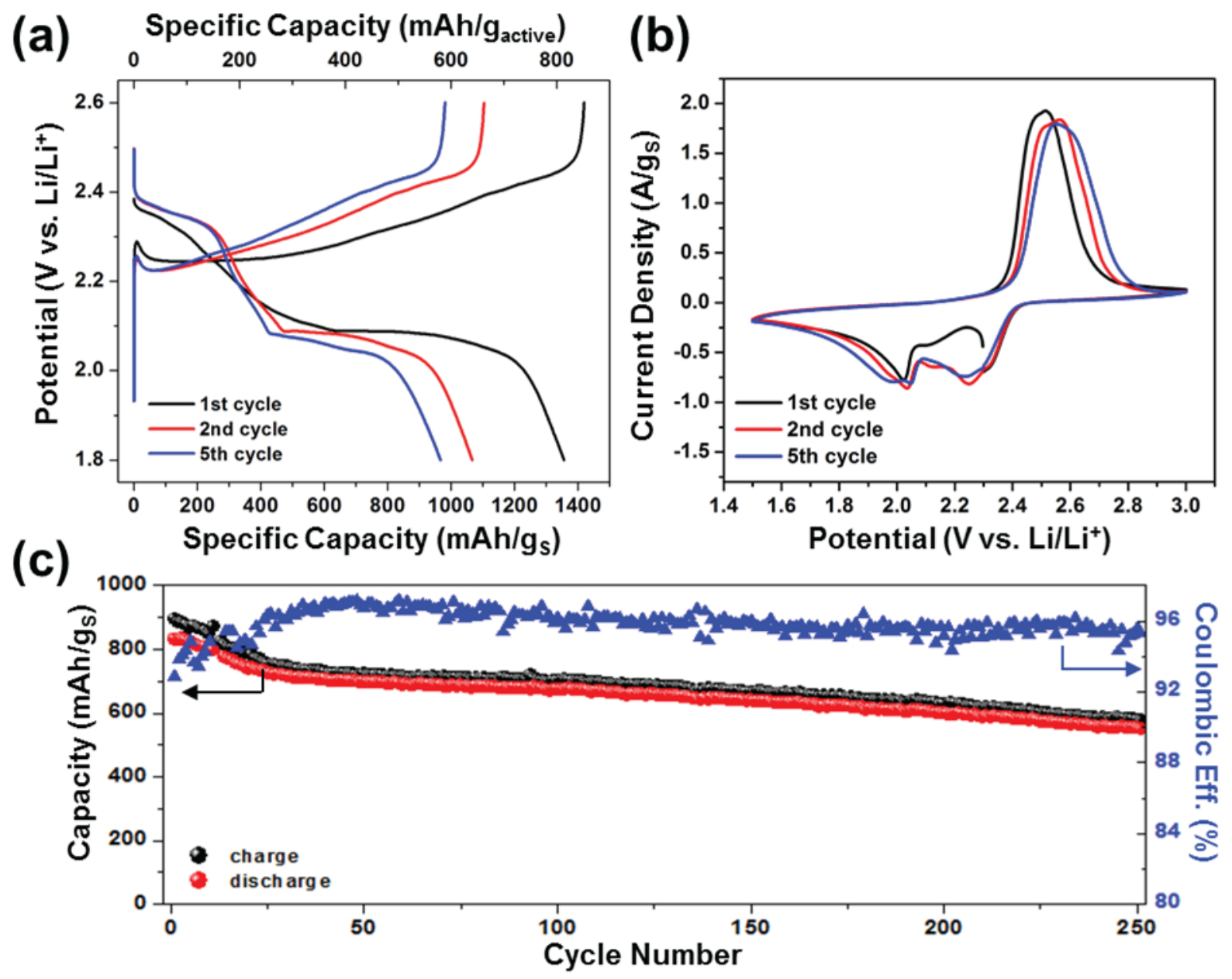

Fig. 4. Electrochemical tests of porous silicon-derived mesoporous carbons as lithium sulfur battery cathodes. (a) galvanostatic charge discharge at $0.1 \mathrm{C}$ rate showing the first five cycles, (b) Cyclic voltammetry at scan rate $0.1 \mathrm{mV} / \mathrm{s}$ for the first five cycles, (c) Durability measurements based on galvanostatic charge discharge at $0.2 \mathrm{C}$ rates over 250 cycles. Blue points correspond to the Coulombic efficiency, labeled on right side axis.

production of the solid low order polysulfides $\left(\mathrm{Li}_{2} \mathrm{~S}_{2}\right.$ and $\left.\mathrm{Li}_{2} \mathrm{~S}\right)$ most likely a result of irreversibly blocked surface area, which is crucial for reversible deposition. Cathodic performance was further probed through cyclic voltammetry at scan rate of $0.1 \mathrm{mV} / \mathrm{s}$. The two-step polysulfide transition[50] is apparent in the CV curves (Fig. 4b) as well as the charge discharge curves (Fig. 
4a). On the initial discharge, the first conversion near $2.3 \mathrm{~V}$ involves the first major transition of $\mathrm{S}_{8}$ to $\mathrm{Li}_{2} \mathrm{~S}_{6}$, while the second peak near $2.1 \mathrm{~V}$ is the complete conversion of the material to $\mathrm{Li}_{2} \mathrm{~S}$. Again in this characterization, the stability of the first peak in subsequent cycles emphasizes the ability of the material to confine polysulfides before dissolution of soluble $\mathrm{S}_{6}{ }^{2-}$ can occur. The initial capacity fade that is manifested in the total area under the anodic and cathodic CV scan is apparent in Fig. 4b as well as a slight degradation between $2^{\text {nd }}$ and $5^{\text {th }}$ cycle. Rate capability of the material is presented in the supporting information (Fig. S10). The capacity retention at fast rates is not remarkable with only $\sim 30 \%$ retention at $5 \mathrm{X}$ current density, which we attribute to the presence of high aspect ratio nanopores in the carbon materials. As these materials are derived from porous silicon templates with highly tunable structural characteristics, we expect optimization of microstructural properties and thickness can improve rate performance and extend the high capacity and excellent cycling stability to be addressable at higher current densities.

From durability experiments that were conducted over 250 cycles at rates of $0.2 \mathrm{C}$, it is evident that a small capacity fade occurs in the first 20 cycles, and then the capacity stabilizes. This initial fade is attributed toa balance of available anchoring sites to retain soluble polysulfides andsurface area to accommodate insoluble $\mathrm{Li}_{2} \mathrm{~S}$ species formed.[48]At this rate67\% of the capacity is retained, meaning the amount of low order polysulfides is slightly decreased. This allows for slower degradation of the carbon host surfaec, as the surface area is better optimized to accommodate the discharge products formed at this rate. This idea is confirmed by the HOPS/LOPS ratio as discussed with Fig. 4a and emphasizing that this initial fade could be mitigated by improving the total surface area of anchoring sites in the porous carbon matrix.From the first to $100^{\text {th }}$ cycle, we observed $81 \%$ capacity retention, which isimpressive in 
comparison to previous efforts in carbon anchoring materials[5, 7, 15-17, 19, 26, 28, 36, 41] and approaches the aim of lithium sulfur chemistries to obtain 90\% retention over 100 cycles.[51] This performance exceeds other very recent (2016) sulfur containing carbon cathodes, specifically those of similar initial discharge and charge current which exhibit capacity retention at 100 cycles of $\sim 72 \%$,[52] $\sim 60 \%$,[53] and $\sim 65 \%[48]$. As many other studies employ inactive anchoring additives or membranes to simultaneously achieve high capacity and stable cycling performance since these are not achieved simultaneously with crystalline carbon materials, we emphasize the promise of this material as a single platform to achieve both high specific performance and stable cycling. We directly attribute this improved performance to the two-fold polysulfide confinement that builds from structural confinement from the porous structure and chemical anchoring due to the oxidized, high defect content carbon surface. As a result, full conversion of $\mathrm{S}_{8}$ to $\mathrm{Li}_{2} \mathrm{~S}$ is achieved on discharge and reversed on the charge. The ability to fully recharge is directly represented in the high Coulombic efficiencies greater than95\%between the $20^{\text {th }}$ to the $250^{\text {th }}$ cycle. In previous studies, maintaining high Coulombic efficiencies and achieving good durability - two closely related features, have presented a major challenge.In this work, we show that this new family of materials that are derived from electrochemically etched templates, can overcome these challenges and enable optimized performance in lithium sulfur battery cathodes.

\section{Conclusions}

In conclusion, we present a new technique to produce lithium sulfur battery electrodes based on coating high defect-containing carbon onto sacrificial porous silicon templates. This yields an oxidized carbon surface demonstrated to be effective for polysulfide anchoring in 
addition to the structural confinement offered by the porous nature of the carbon. Combined, this enables us to achieve initial capacity of $1350 \mathrm{mAh} / \mathrm{g}_{\text {sulfurat }} 0.1 \mathrm{C}$ and81\% capacity retention over 100 cycles at slow rates of $0.2 \mathrm{C}$, which is competitive or better than the best reported stateof-the-art cathode materials. Compared to other routes, structural control enabled from the porous silicon template builds on over six decades of research in low-cost semiconductor manufacturing and is transformed into a sacrificial template for defect-controlled porous carbons through scalable and low-cost steps such as acetylene treatment and dissolution in (basic) salt water. Unlike other routes to produce mesoporous carbons, the independent control over (1) the structure of the porous silicon and (2) the chemical properties of the carbon grown on the porous silicon enables a fine control knob on tailoring the chemical and structural properties to be optimized for sulfur cathodes. The highly tunable features leave extensive room to optimize the material surface area for enhanced capacity and pore structure for enhanced rate capability. This opens an exciting area to connect fabrication processes rooted in low-cost semiconductor manufacturing with emerging battery systems that have promise to advance beyond the performance of lithium-ion batteries.

\section{Acknowledgement}

We are grateful to Landon Oakes, Mengya Li, and Andrew Westover for helpful discussions, Rizia Bardhan for use of Raman spectrometer and UV/vis spectrometer, and Dmitry Koktysh for assistance with TGA and FTIR measurements. This work was supported by NSF CMMI grant 1334269, and A.D., K.S., and A.P.C. were supported in part by the National Science Foundation Graduate Research Fellowship under Grant No. 1445197. 


\section{References}

[1] P.G. Bruce, S.A. Freunberger, L.J. Hardwick, J.M. Tarascon, Nat Mater, 11 (2012) 19-29.

[2] H.J. Peng, Q. Zhang, Angew Chem Int Edit, 54 (2015) 11018-11020.

[3] Z.W. Seh, W.Y. Li, J.J. Cha, G.Y. Zheng, Y. Yang, M.T. McDowell, P.C. Hsu, Y. Cui, Nat Commun, 4 (2013) 1331.

[4] X.L. Ji, K.T. Lee, L.F. Nazar, Nat Mater, 8 (2009) 500-506.

[5] C.D. Liang, N.J. Dudney, J.Y. Howe, Chem Mater, 21 (2009) 4724-4730.

[6] S.R. Chen, Y.P. Zhai, G.L. Xu, Y.X. Jiang, D.Y. Zhao, J.T. Li, L. Huang, S.G. Sun, Electrochim Acta, 56 (2011) 9549-9555.

[7] X.L. Li, Y.L. Cao, W. Qi, L.V. Saraf, J. Xiao, Z.M. Nie, J. Mietek, J.G. Zhang, B.

Schwenzer, J. Liu, J Mater Chem, 21 (2011) 16603-16610.

[8] G.Y. Zheng, Y. Yang, J.J. Cha, S.S. Hong, Y. Cui, Nano Lett, 11 (2011) 4462-4467.

[9] J. Scheers, S. Fantini, P. Johansson, J Power Sources, 255 (2014) 204-218.

[10] H.B. Yao, K. Yan, W.Y. Li, G.Y. Zheng, D.S. Kong, Z.W. Seh, V.K. Narasimhan, Z. Liang, Y. Cui, Energ Environ Sci, 7 (2014) 3381-3390.

[11] R. Singhal, S.H. Chung, A. Manthiram, V. Kalra, J Mater Chem A, 3 (2015) 4530-4538.

[12] L. Qie, A. Manthiram, ACS Energy Lett., 1 (2016) 46-51.

[13] Z.W. Seh, W.Y. Li, J.J. Cha, G.Y. Zheng, Y. Yang, M.T. McDowell, P.C. Hsu, Y. Cui, Nat Commun, 4 (2013).

[14] W.D. Zhou, Y.C. Yu, H. Chen, F.J. DiSalvo, H.D. Abruna, J Am Chem Soc, 135 (2013) 16736-16743.

[15] S.K. Liu, K. Xie, Y.J. Li, Z.X. Chen, X.B. Hong, L.J. Zhou, J.F. Yuan, C.M. Zheng, Rsc Adv, 5 (2015) 5516-5522.

[16] H.L. Wang, Y. Yang, Y.Y. Liang, J.T. Robinson, Y.G. Li, A. Jackson, Y. Cui, H.J. Dai, Nano Lett, 11 (2011) 2644-2647.

[17] G.L. Xu, Y.F. Xu, J.C. Fang, X.X. Peng, F. Fu, L. Huang, J.T. Li, S.G. Sun, Acs Appl Mater Inter, 5 (2013) 10782-10793.

[18] G.Y. Xu, B. Ding, P. Nie, L.F. Shen, H. Dou, X.G. Zhang, Acs Appl Mater Inter, 6 (2014) 194-199.

[19] C. Hoffmann, S. Thieme, J. Bruckner, M. Oschatz, T. Biemelt, G. Mondin, H. Althues, S. Kaskel, Acs Nano, 8 (2014) 12130-12140.

[20] H.J. Peng, J.Y. Liang, L. Zhu, J.Q. Huang, X.B. Cheng, X.F. Guo, W.P. Ding, W.C. Zhu, Q. Zhang, Acs Nano, 8 (2014) 11280-11289.

[21] Q. Sun, B. He, X.Q. Zhang, A.H. Lu, Acs Nano, 9 (2015) 8504-8513.

[22] J.T. Lee, Y.Y. Zhao, S. Thieme, H. Kim, M. Oschatz, L. Borchardt, A. Magasinski, W.I.

Cho, S. Kaskel, G. Yushin, Adv Mater, 25 (2013) 4573-4579.

[23] N. Jayaprakash, J. Shen, S.S. Moganty, A. Corona, L.A. Archer, Angew Chem Int Edit, 50

(2011) 5904-5908.

[24] J.L. Shi, H.J. Peng, L. Zhu, W.C. Zhu, Q. Zhang, Carbon, 92 (2015) 96-105.

[25] R. Sahore, L.P. Estevez, A. Ramanujapuram, F.J. DiSalvo, E.P. Giannelis, J Power Sources, 297 (2015) 188-194.

[26] Q.F. Zhang, Y.P. Wang, Z.W. Seh, Z.H. Fu, R.F. Zhang, Y. Cui, Nano Lett, 15 (2015) 3780-3786.

[27] Y.H. Xu, Y. Wen, Y.J. Zhu, K. Gaskell, K.A. Cychosz, B. Eichhorn, K. Xu, C.S. Wang, Adv Funct Mater, 25 (2015) 4312-4320. 
[28] B. Zhang, X. Qin, G.R. Li, X.P. Gao, Energ Environ Sci, 3 (2010) 1531-1537.

[29] S. Xin, L. Gu, N.H. Zhao, Y.X. Yin, L.J. Zhou, Y.G. Guo, L.J. Wan, J Am Chem Soc, 134 (2012) 18510-18513.

[30] M. Helen, M.A. Reddy, T. Diemant, U. Golla-Schindler, R.J. Behm, U. Kaiser, M. Fichtner, Sci Rep, 5 (2015) 12146.

[31] G.M. Zhou, Y.B. Zhao, C.X. Zu, A. Manthiram, Nano Energy, 12 (2015) 240-249.

[32] X. Liang, C. Hart, Q. Pang, A. Garsuch, T. Weiss, L.F. Nazar, Nat Commun, 6 (2015) 5682.

[33] Q. Pang, D. Kundu, M. Cuisinier, L.F. Nazar, Nat Commun, 5 (2014) 4759.

[34] H.B. Yao, G.Y. Zheng, P.C. Hsu, D.S. Kong, J.J. Cha, W.Y. Li, Z.W. Seh, M.T. McDowell, K. Yan, Z. Liang, V.K. Narasimhan, Y. Cui, Nat Commun, 5 (2014) 3943.

[35] W.D. Zhou, C.M. Wang, Q.L. Zhang, H.D. Abruna, Y. He, J.W. Wang, S.X. Mao, X.C.

Xiao, Adv Energy Mater, 5 (2015) 1401752.

[36] L.W. Ji, M.M. Rao, H.M. Zheng, L. Zhang, Y.C. Li, W.H. Duan, J.H. Guo, E.J. Cairns, Y.G. Zhang, J Am Chem Soc, 133 (2011) 18522-18525.

[37] R. Carter, S. Chatterjee, E. Gordon, K. Share, W.R. Erwin, A.P. Cohn, R. Bardhan, C.L. Pint, Nanoscale, 7 (2015) 16755-16762.

[38] S. Chatterjee, R. Carter, L. Oakes, W.R. Erwin, R. Bardhan, C.L. Pint, J Phys Chem C, 118 (2014) 10893-10902.

[39] L. Oakes, A. Westover, J.W. Mares, S. Chatterjee, W.R. Erwin, R. Bardhan, S.M. Weiss, C.L. Pint, Sci Rep, 3 (2013) 3020.

[40] A.P. Cohn, W.R. Erwin, K. Share, L. Oakes, A.S. Westover, R.E. Carter, R. Bardhan, C.L. Pint, Nano Lett, 15 (2015) 2727-2731.

[41] C.M. Xu, Y.S. Wu, X.Y. Zhao, X.L. Wang, G.H. Du, J. Zhang, J.P. Tu, J Power Sources, 275 (2015) 22-25.

[42] M. Hagen, D. Hanselmann, K. Ahlbrecht, R. Maca, D. Gerber, J. Tubke, Adv Energy Mater, 5 (2015) 1401986.

[43] Q.C. Zeng, D.W. Wang, K.H. Wu, Y. Li, F.C. de Godoi, I.R. Gentle, J Mater Chem A, 2 (2014) 6439-6447.

[44] L. Stobinski, B. Lesiak, L. Kover, J. Toth, S. Biniak, G. Trykowski, J. Judek, J Alloy Compd, 501 (2010) 77-84.

[45] D. Pantea, H. Darmstadt, S. Kaliaguine, L. Summchen, C. Roy, Carbon, 39 (2001) 11471158.

[46] R. Sahore, B.D.A. Levin, M. Pan, D.A. Muller, F.J. DiSalvo, E.P. Giannelis, Adv. Energy Mater., (2016) 1614-6840.

[47] C. Barchasz, F. Molton, C. Duboc, J.C. Lepretre, S. Patoux, F. Alloin, Anal Chem, 84 (2012) 3973-3980.

[48] Y.J. Wei, Y.Q. Tao, C.F. Zhang, J.T. Wang, W.M. Qiao, L.C. Ling, D.H. Long, Electrochim Acta, 188 (2016) 385-392.

[49] J. Park, J. Moon, C. Kim, J.H. Kang, E. Lim, J. Park, K.J. Lee, S.-H. Yu, J.-H. Seo, J. Lee, J. Heo, N. Tanaka, S.-P. Cho, J. Pyun, J. Cabana, B.H. Hong, Y.-E. Sung, NPG Asia Mater., 8 (2016) 272.

[50] Y.S. Su, Y.Z. Fu, T. Cochell, A. Manthiram, Nat Commun, 4 (2013) 2984.

[51] S. Evers, L.F. Nazar, Acc. Chem. Res., 46 (2013) 1135-1143.

[52] X.B. Yang, W. Zhu, G.B. Cao, X.D. Zhao, Rsc Adv, 6 (2016) 7159-7171.

[53] L. Sun, D.T. Wang, Y.F. Luo, W.B. Kong, Y. Wu, L.N. Zhang, K.L. Jiang, Q.Q. Li, Y.H. Zhang, J.P. Wang, S.S. Fan, Acs Nano, 10 (2016) 1300-1308. 
Porous Silicon Templated Carbon - High Porosity

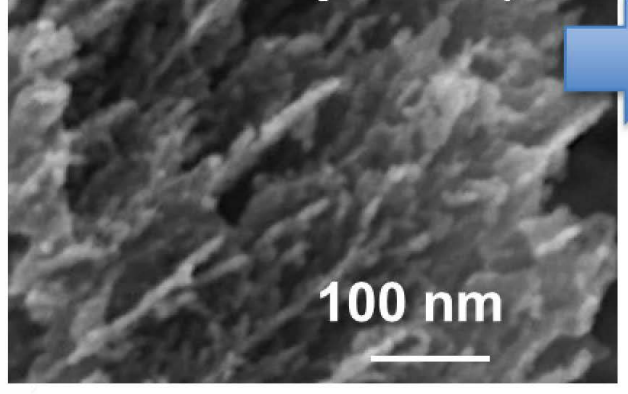

3-D Carbon Network: Polysulfide Confinement
Oxidized Carbons: Polysulfide Anchoring

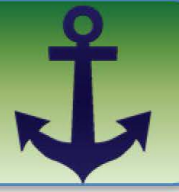

soluble Polysulfide

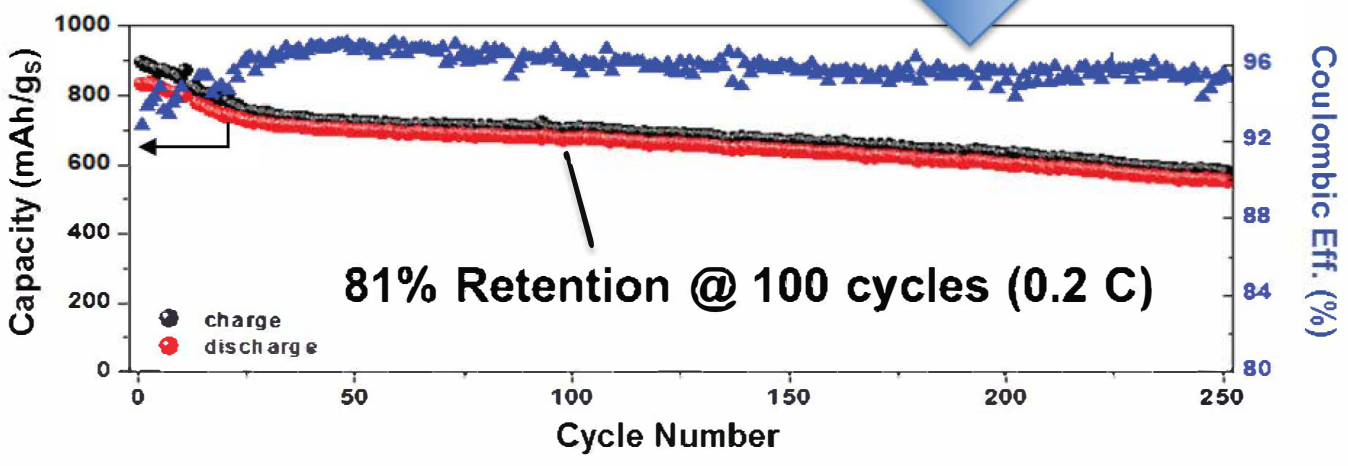

